10 - ORIGINAL ARTICLE

CLINICAL INVESTIGATION

\title{
Comparison between hemosiderin and Technetium-99 in sentinel lymph node biopsy in human breast cancer ${ }^{1}$
}

\author{
Paulo Henrique Diógenes Vasques ${ }^{\mathrm{I}}$, Mayara Maia Alves ${ }^{\mathrm{II}}$, Ranniere Gurgel Furtado de Aquino ${ }^{\mathrm{III}}$, Roberto Vitor Almeida Torres ${ }^{\mathrm{IV}}$, \\ José Lucas Martins Bezerra ${ }^{\mathrm{IV}}$, Luis Porto Brasileirov', Luiz Gonzaga Porto Pinheiro ${ }^{\mathrm{VI}}$
}

DOI: http://dx.doi.org/10.1590/S0102-865020150110000010

IFellow PhD degree, Postgraduate Program in Surgery, Department of Surgery, Universidade Federal do Ceará (UFC), Fortaleza-CE, Brazil. Conception and design of the study, acquisition of data, manuscript writing.

IIFellow PhD degree, Postgraduate Program in Biotechnology, Renorbio, UFC, Fortaleza-CE, Brazil. Conception and design of the study, acquisition of data, manuscript writing.

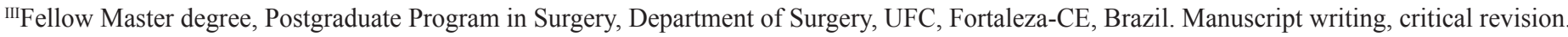

${ }^{\mathrm{IV}}$ Graduate student, Medicine Faculty, UFC, Fortaleza-CE, Brazil. Acquisition of data.

${ }^{v}$ Graduate student, Medicine Faculty, INTA, Sobral-CE, Brazil. Acquisition of data.

${ }^{\mathrm{VI}}$ Chairman, Full Professor, Department of Surgery, Medicine Faculty, UFC, Fortaleza-CE, Brazil. Intellectual, scientific, conception and design of the study.

\section{ABSTRACT}

PURPOSE: To assess the safety and potential equivalence of the use of hemosiderin compared to the Technetium-99 in sentinel lymph node biopsy in human breast cancer.

METHODS: Non-random sample of 14 volunteer women diagnosed with breast cancer with primary tumors (T1/T2) and clinically tumor-free axilla were submitted to the identification of sentinel lymph node using hemosiderin obtained from autologous blood injected in the periareolar region $24 \mathrm{~h}$ before surgery on an outpatient basis. Patients received preoperative subareolar intradermal injection of Technetium-99 in the immediate preoperative period. Patients were submitted to sentinel lymph node biopsy, with incision in the axillary fold guided by Gamma-Probe, dissection by planes until the identification of the point of maximum uptake of Technetium-99, identifying the marked nodes and their colors. All surgical specimens were sent for pathological and immunohistochemical study.

RESULTS: The results showed no evidence of side effects and/or allergic and non-allergic reactions in patients submitted to SLNB with hemosiderin. The SLN identification rate per patient was $100 \%$. SLNB identification rate per patient with hemosiderin was the same as that of Technetium, with a concordance rate of $100 \%$ between the methods.

CONCLUSION: Hemosiderin is a safe dye that is equivalent to Technetium in breast sentinel lymph node biopsy.

Key words: Breast Neoplasms. Sentinel Lymph Node Biopsy. Hemosiderin. Technetium. 


\section{Introduction}

The Sentinel Lymph Node (SLN) is the first lymph node to receive lymphatic drainage from the site of a primary tumor, a concept established since 1977 by Cabanas, who introduced the sentinel lymph node biopsy (SLNB) in penile carcinoma ${ }^{1}$. In 1993, Krag et $a l .{ }^{2}$ used the technetium-99 $\left({ }^{99} \mathrm{Tc}\right)$ in SLNB for melan. In 1994, Giuliano, using the patent blue dye in breast tumor, validated the concept of sentinel lymph node (SLN) in breast cancer ${ }^{3}$. In 2003, Veronesi ${ }^{4}$ established that SLNB was a safe and accurate method of screening axillary nodes for metastasis in women with small breast tumors. Recently, SLNB has replaced axillary dissection in early-stage breast cancer in clinically tumor-free patients $^{5}$.

The association of ${ }^{99} \mathrm{Tc}$ with the blue dye allowed a more accurate identification in $\mathrm{SLNB}^{6,7}$. Other dyes can also be used in SLN identification during the surgical procedure, such as methylene blue, patent blue, and isosulfan blue ${ }^{8}$ however, such substances - in a recent literature review - revealed a significant number of hypersensitivity reactions ${ }^{9,10}$. Patent blue dye can cause from subtle adverse effects, such as skin rash, to severe reactions of anaphylaxis ${ }^{11,12}$. The use of methylene blue may be associated with serious complications such as skin and fat necrosis at the injection site $^{13}$. Anaphylactic reactions to isosulfan blue or patent blue in patients submitted to SLNB range from $0.6 \%$ to $2.7 \%{ }^{14}$.

Identifying substances with fewer side effects and/ or allergic reactions in SLNB is a challenge. In 2009, Pinheiro et $a l .{ }^{15}$ proved, in an experimental research with animals, the efficacy of hemosiderin (a protein resulting from the degradation of hemoglobin that is commonly found in lysosomes of histiocytes and Kupffer cells) as an autologous dye in SLNB of female dogs' breasts. In the present study, the association of hemosiderin with ${ }^{99} \mathrm{Tc}$ presented similar results in the SLNB when compared to patent blue and ${ }^{99} \mathrm{Tc}$. Hemosiderin has been experimentally proven to be a potential alternative to the current dyes.

The purpose of the present research was to assess the safety and potential equivalence of the use of hemosiderin in SLNB in human breast cancer compared to the gold standard test, the ${ }^{99} \mathrm{Tc}$.

\section{Methods}

This non-randomized prospective clinical study conducted with human subjects was approved by the Research Ethics Committee of the Hospital Universitário Walter Cantídio, Universidade Federal do Ceará on August 10, 2010, No. 0730710. The surgical procedures were carried out at the Maternidade Escola Assis Chateaubriand - MEAC and Hospital Universitário Walter Cantídio by one single surgeon in the period from September 2011 to November 2013.
The non-random sample comprised 14 women from the phase 1 clinical trial who were selected in the mastology outpatient clinic of the MEAC and had a confirmed histopathologic diagnosis of breast cancer, with primary tumors (T1/T2) and clinically tumor-free axilla; women were of different ages, weighted over 50 $\mathrm{kg}$, and had an indication for SLNB (Table 1).

Each patient was informed about the procedures and signed a free informed consent form prior to research participation. A week before the start of the study, all the participants underwent preoperative clinical and laboratory assessment, which showed they were able to undergo the surgical procedure. The iron metabolic profile was also assessed previously through complete blood count, serum iron, serum ferritin, transferrin saturation, and total iron-binding capacity.

\section{Preparation, administration and production of he- mosiderin}

The preparation of the autologous dye derived from blood - hemosiderin - for use in the present study was obtained from a sample of $16 \mathrm{ml}$ of peripheral blood collected $24 \mathrm{~h}$ before surgery. The peripheral blood drawn from the patient was inserted into two BD Vacutainer ${ }^{\circledR}$ tubes with Buffered Sodium Citrate aseptically. Then, the blood was centrifuged at $2000 \mathrm{rpm}$ for 10 minutes at $22^{\circ} \mathrm{C}$. The centrifuged material separated into three phases in the test tube. The upper and intermediate layers (serum) were discarded and the bottom layer (red blood cells - RBCs) was diluted with normal saline $(0.9 \% \mathrm{NaCl})$ in equal volume $(\mathrm{ml})$ followed by manual homogenization in laminar flow biological safety cabinet. The resulting solution was centrifuged (3800 rpm for 3 minutes) again, with the appearance of two phases the first was discarded, and the volume withdrawn was replaced with double-distilled water in the laminar flow cabinet, causing hemolysis of RBCs. The third and final centrifugation (3800 rpm for 3 minutes) yield one single phase of lysed RBCs suspended in the reddish liquid. This hemolysate, rich in hemoglobin, which contains iron, when injected in the breast is phagocytosed by macrophages of the Reticuloendothelial System that, through the lymphatic system, saturate the sentinel lymph node of ferric hydroxyphosphate micelles, which accumulate, resulting in the formation of hemosiderin. Sterility assurance was controlled with blood cultures to detect bacteria and fungi.

Hemosiderin was injected on an outpatient basis, with the patient in supine position, asepsis of the injection site with $70 \%$ alcohol, local anesthesia with $2 \%$ lidocaine without adrenaline, in the external periareolar region of the breast (3h) via a single puncture. After that, we performed a subareolar injection of $4 \mathrm{ml}$ of the dye and, after removal of the needle, we pressed the puncture hole with cotton for 1 minute (Figure 1A-B) 
Patients were hospitalized and assessed as for the presentation of side effects for 24 hours. Two hours before the surgical procedure, the patient was taken to the imaging center of the clinic and underwent breast Magnetic Resonance Imaging (MRI) and received preoperative subareolar intradermal injection of $0.2 \mathrm{ml}$ of ${ }^{99} \mathrm{Tc}$ and mammary scintigraphy. After imaging tests, the patients were taken to the operating room where they, under general anesthesia, underwent the surgical procedure.

\section{Sentinel lymph node biopsy}

Patients were submitted to SLNB, with incision in the axillary fold guided by Gamma-Probe and dissection by planes until the identification of point of maximum uptake of ${ }^{99} \mathrm{Tc}$, identifying the marked nodes and their colors (Figure 1C-D). After removal of lymph nodes, we assessed in each one the detection intensity of ${ }^{99} \mathrm{Tc}$ by the gamma-probe and the hemosiderin level of marking using a color scale from red to black (Figure 2). These data were recorded in a data collection form for further analysis. All surgical specimens were sent for pathological and immunohistochemical study. The patients were monitored throughout the procedure, since the injection of hemosiderin until the postoperative period and their return within 7, 15 and 30 days after surgery (Figure 3).
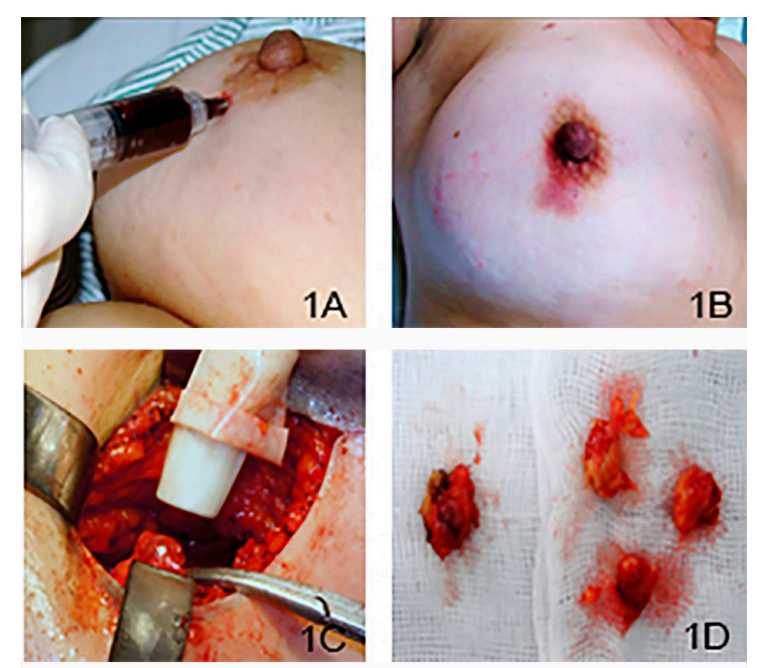

FIGURE 1 - Procedures: (A) Subareolar injection of hemosiderin. (B) Site after injection of hemosiderin (small bruise). (C) Identification of sentinel lymph node with the Gamma-Probe. (D) Sentinel lymph nodes marked with hemosiderin.
2A

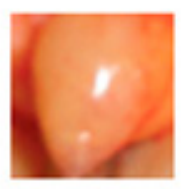

$2 \mathrm{~B}$

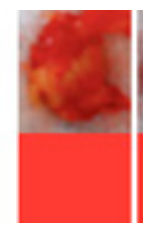

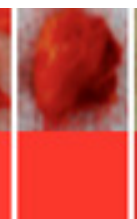

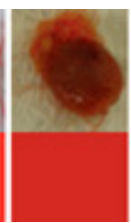

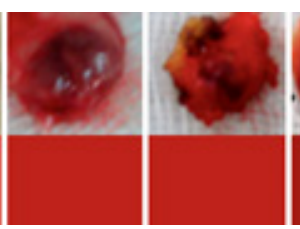
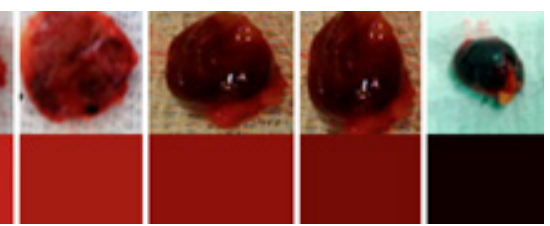

(B) Lymph node marked with hemosiderin and the FIGURE 2 - Macroscopic aspect of the SLN color: (A) Ly
comparative scale of color intensity ranging from red to black.

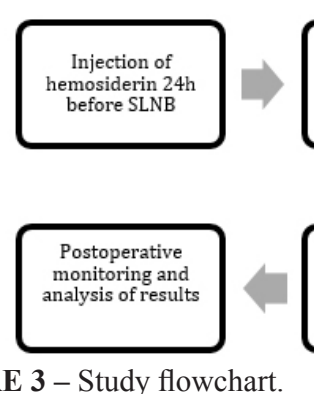

FIGURE 3 - Study flowchart.

\section{Results}

Fourteen patients underwent SLNB with hemosiderin according to the method described. The mean age of patients was 54.3 years and the postmenopausal status was prevalent in $50 \%$ of the sample. Regarding histological type, twelve patients (85\%) were diagnosed with invasive ductal carcinoma. As for the histological grade, there was a predominance of grade 2 tumors $(60 \%)$ (Table 1$)$.

There was no evidence of side effects, allergic reactions, surgical infection or toxicity in patients submitted to SLNB with hemosiderin in our study.

The efficacy of SLNB with hemosiderin was confirmed by determining the equivalence of hemosiderin compared to the marking technique using ${ }^{99} \mathrm{Tc}$, which revealed a similar detection of SLN marked with hemosiderin and ${ }^{99} \mathrm{Tc}$ in all the patients. The SLN identification rate was $100 \%$, with an average of 2.5 SLN found per patient. The study identified the SLN marked with hemosiderin in $100 \%$ of cases (Figure 4 ). 
TABLE 1 - Patient and tumor characteristics of the 14 patients included. Results are given as mean values or frequencies in $\%$.

\begin{tabular}{|c|c|}
\hline Characteristic & Value \\
\hline Age & 54.3 y (range $36-85$ y) \\
\hline Height & $156.4 \mathrm{~cm}$ (range $143-172 \mathrm{~cm})$ \\
\hline Weight & $68.4 \mathrm{~kg}$ (range $48-88 \mathrm{~kg})$ \\
\hline Body mass index (BMI) & 27.8 (range 22.1 - 33.5) \\
\hline \multicolumn{2}{|l|}{ Menopausal status } \\
\hline Premenopause & $4(28 \%)$ \\
\hline Perimenopause & $3(22 \%)$ \\
\hline Postmenopause & $7(50 \%)$ \\
\hline \multicolumn{2}{|l|}{ Carcinoma type } \\
\hline Invasive carcinoma & $12(85 \%)$ \\
\hline Ductal carcinoma in situ & $2(15 \%)$ \\
\hline \multicolumn{2}{|l|}{ Tumor location } \\
\hline Upper-outer quadrant & $6(42 \%)$ \\
\hline Upper-inner quadrant & $2(14 \%)$ \\
\hline Lower-inner quadrant & $2(14 \%)$ \\
\hline Lower-outer quadrante & $4(30 \%)$ \\
\hline Central & $0(0 \%)$ \\
\hline \multicolumn{2}{|l|}{ Pathological tumor size } \\
\hline $\mathrm{pTis}$ & $2(15 \%)$ \\
\hline pT1a & $1(7 \%)$ \\
\hline pT1b & $0(0 \%)$ \\
\hline pT1c & $6(43 \%)$ \\
\hline pT2 & $5(35 \%)$ \\
\hline pT3 & $0(0 \%)$ \\
\hline \multicolumn{2}{|c|}{ Pathological lymph node status } \\
\hline pN0 & $10(72 \%)$ \\
\hline pNi. & $0(0 \%)$ \\
\hline $\mathrm{pN} 1 \mathrm{mi}$ & $1(7 \%)$ \\
\hline pN1a & $2(14 \%)$ \\
\hline $\mathrm{pN} 1 \mathrm{~b}$ & $1(7 \%)$ \\
\hline $\mathrm{pN} 2 \mathrm{a}$ & $0(0 \%)$ \\
\hline \multicolumn{2}{|l|}{ Grading } \\
\hline G1 & $2(16.7 \%)$ \\
\hline $\mathrm{G} 2$ & $9(60 \%)$ \\
\hline G3 & $3(23.3 \%)$ \\
\hline \multicolumn{2}{|l|}{ Hormone receptor statu } \\
\hline Estrogen receptor (ER). & $12(86 \%)$ \\
\hline Progesterone receptor (PR). & $12(86 \%)$ \\
\hline \multicolumn{2}{|l|}{ HER2 status } \\
\hline HER2. & $0(0 \%)$ \\
\hline
\end{tabular}
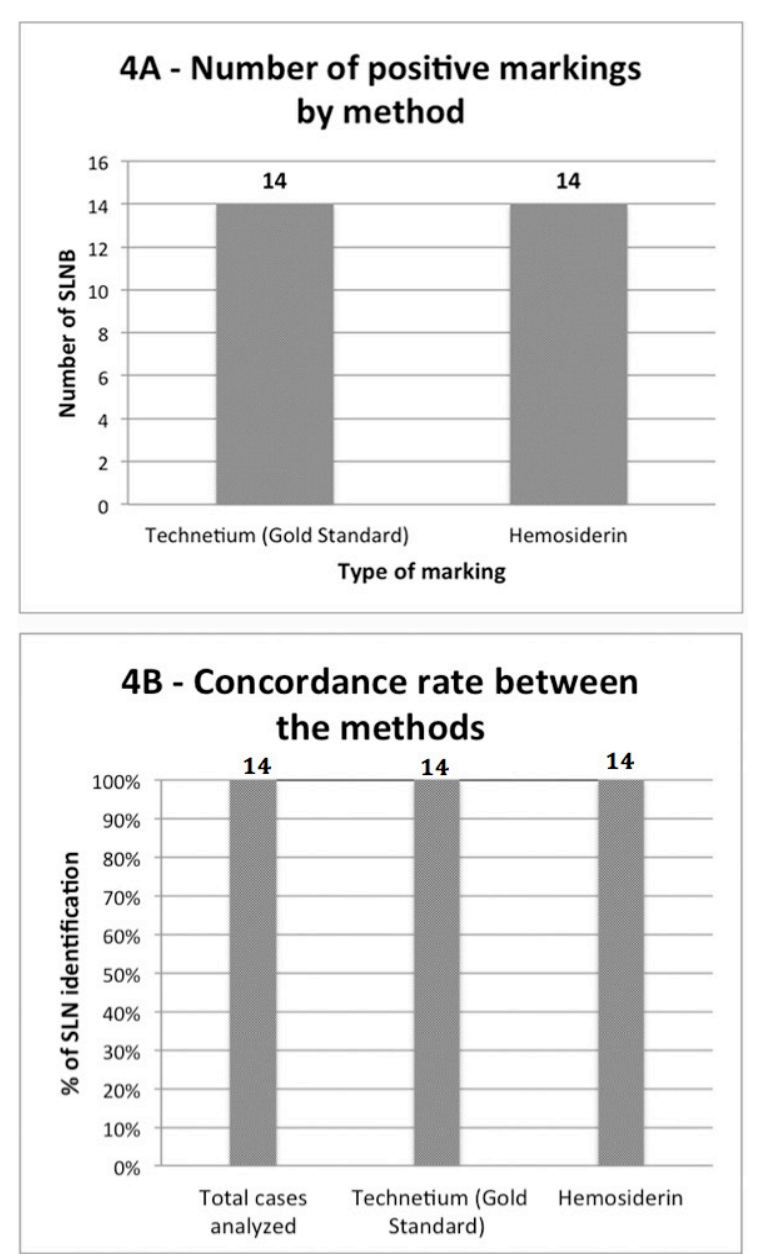

FIGURE 4 - (A) SLN marked with hemosiderin and ${ }^{99}$ Tc per patients*. (B) Concordance rate between hemosiderin and ${ }^{99} \mathrm{Tc}$. *The average number of SLN found per patients was 2.5.

Each patient submitted to SLNB had their lymph nodes classified in percentage terms according to the shades of color found: no color- $0 \%(\mathrm{n}=0)$; light red-16\% $(\mathrm{n}=2)$; dark-red $64 \%$ $(\mathrm{n}=8)$ and black-16\% $(\mathrm{n}=2)$ (Table 2$)$. In $100 \%$ of cases $(\mathrm{n}=14)$, the SLN were marked and identified by gamma-probe with ${ }^{99} \mathrm{Tc}$.

TABLE 2 - Shades of color of lymph nodes.

\begin{tabular}{cc}
\hline Color of Lymph Node/patients & Frequency \\
\hline No color & $0(0 \%)$ \\
Light red & $2(16 \%)$ \\
Dark red & $8(64 \%)$ \\
Black & $2(16 \%)$ \\
\hline
\end{tabular}

\section{Discussion}

Currently, the gold standard in the SLNB is the use of radiotracer ${ }^{99} \mathrm{Tc} 99 /$ gamma-probe technique in combination with blue dye ${ }^{7}$. Disadvantages of these techniques, as well as the 
publication of adverse effects and allergic reactions associated with dyes, which are life threatening, have led to new research as an alternative to improve and even substitute existing methods.

The use of blue dyes for SLNB has increased worldwide. Sleth et al. ${ }^{16}$, in their work, have reported an annual increase in the incidence of adverse reactions and increased risk of allergic reactions associated with the blue dyes; therefore, they questioned if it was not the time to change the dye. This restlessness was one of the motivations to study hemosiderin - in the present clinical study - as a dye that can substitute artificial dyes for SLNB.

Pinheiro et al. ${ }^{15}$ have tested the feasibility of hemosiderin in laboratory as an autologous dye, compared to patent blue, for breast SLNB in a canine model. Given that original study, it was possible to base and test the use of hemosiderin for SLNB in breast cancer in women, revealing an equivalence of hemosiderin and ${ }^{99} \mathrm{Tc}$ with a concordance rate of $100 \%$ of the cases and an average of 2.5 SLN per patients. These findings put hemosiderin on the same level of ${ }^{99} \mathrm{Tc}$, a widely used substance that is currently the "gold standard" in SLNB worldwide?.

As this is a phase I clinical trial with a sample of 14 women and with the aim to establish the clinical safety of the substance tested and the potential equivalence to ${ }^{99} \mathrm{Tc}$, its findings open a promising direction for new studies on the use of hemosiderin in the SLNB in breast cancer with larger samples and the possibility to extend its indication to other tumor sites that employ the SLNB.

In the present study, hemosiderin was administered 24 hours before the procedure, as recommended by Pinheiro et al. ${ }^{15}$, who demonstrated that this interval allowed a stronger coloring of SLN. This interval has enabled the monitoring of possible reactions during the administration of the substance tested as well as in the preoperative hospital stay. As it is an autologous dye, no signs or symptoms of allergies were observed during the administration of hemosiderin in the perioperative and/or postoperative period. This fact places hemosiderin as a safe dye, unlike the blue dyes, which are reported by several studies as presenting allergic reactions in SLNB, with an estimated prevalence of $0.6 \%-2.7 \% 0^{6,10,17-20}$. These reactions, in addition to putting patients' lives at risk, can hinder and complicate anesthesia, requiring from the medical staff therapeutic and resuscitative measures that, depending on the severity of the condition, may increase surgical duration, cancel the surgical procedure, and even lead to death. Therefore, the safety of hemosiderin can decrease personal and health spendings, reduce the morbidity of the procedure and emotional suffering, along with other indirect advantages ${ }^{6,10,17-20}$.

Non-allergic reactions associated with the blue dyes are complications that add difficulties and consequences to the
SLNB procedure. The main ones are: interference with pulse oximetry, bluish pigmentation of skin or bodily fluids, and skin necrosis ${ }^{21}$. Unwanted tattoos in the skin with the use of subdermal or intradermal injection technique due to the use of blue dyes are complications described in SLNB. This can be considered a cosmetic problem for women, especially if they persist for many months or years. Studies have shown that this condition persisted in $41 \%$ of patients for 1 year in the postoperative period ${ }^{18}$. The rate of skin pigmentation decreases more rapidly after the second year, but it may remain after three years ${ }^{21,22}$.

The use of hemosiderin, confronting the literature data on non-allergic reactions to blue dyes, did not cause persistent pigmentation of the skin or bodily fluids, or interference with pulse oximetry, or cases of skin necrosis. In some cases, there is a persistence of small areas (ecchymosis and/or bruises) at the site of the subareolar injection, which become unnoticeable in a few days or weeks and disappear without leaving marks and/or permanent sequelae.

In the current scenario of breast cancer treatment, breastconserving and oncoplastic surgeries, with the preservation of breast skin, gradually tend to substitute mastectomies and mutilating surgeries. The presence of tattoos and persistent pigmentations on the breasts due to the use of blue dyes add an anti-aesthetic component that hinder the result of minimally aggressive treatment efforts of modern surgery. Hemosiderin, as demonstrated in the present study, temporarily marks the breast skin with small areas of ecchymosis and/or bruises, allowing better aesthetic results without the unwanted persistent blue tattoos.

The postoperative monitoring of patients of up to 30 days after surgery allowed to evaluate the absence of signs and/ or symptoms of surgical infection in the study patients as well as the results of cultures obtained from hemosiderin samples used in patients, which were negative, confirming the safety of the method and the absence of infectious complications.

In vitro studies indicate the genotoxicity of blue dyes and the increased level of oxidative DNA lesions. The methylene blue and patent blue, commonly used in SLNB, have genotoxic effects on breast cells in clinically relevant concentrations in vitro. In vivo studies are now needed to assess the damage to DNA caused by these dyes during SLNB. Although it is an in vitro study, these substances are not innocuous, as it has been thought ${ }^{23}$. In contrast, hemosiderin, a dye without toxicity derived from the patient, minimizes these concerns.

With the present study, hemosiderin opens a new perspective to become an important tool in SLNB. Further studies with larger samples and consistent results are needed to confirm its 
efficacy and safety. Therefore, it has the potential to substitute the current dyes and become the "gold standard" for SLNB.

\section{Conclusion}

Hemosiderin is an efficient and safe dye that is equivalent to ${ }^{99} \mathrm{Tc}$ in sentinel lymph node biopsy in breast cancer.

\section{References}

1. Cabanas RM. An approach for the treatment of penile carcinoma. Cancer. 1977;39(2):456-66. PMID: 837331.

2. Krag DN, Weaver DL, Alex JC, Fairbank JT. Surgical resection and radiolocalization of the sentinel lymph node in breast cancer using a gamma probe. Surg Oncol. 1993;2(6):335-9; discussion 340. PMID: 8130940.

3. Giuliano AE, Kirgan DM, Guenther JM, Morton DL. Lymphatic mapping and sentinel lymphadenectomy for breast cancer. Ann Surg. 1994;220(3):391-8; discussion 398-401. PMID: 8092905.

4. Veronesi U, Paganelli G, Viale G, Luini A, Zurrida S, Galimberti V, Intra M, Veronesi P, Robertson C, Maisonneuve P, Renne G, De Cicco C, De Lucia F, Gennari R. A randomized comparison of sentinel-node biopsy with routine axillary dissection in breast cancer. N Engl J Med. 2003;349(6):546-53. PMID: 12904519.

5. Lyman GH, Temin S, Edge SB, Newman LA, Turner RR, Weaver DL, Benson AB, 3rd, Bosserman LD, Burstein HJ, Cody H, 3rd, Hayman J, Perkins CL, Podoloff DA, Giuliano AE, American Society of Clinical Oncology Clinical P. Sentinel lymph node biopsy for patients with early-stage breast cancer: American Society of Clinical Oncology clinical practice guideline update. J Clin Oncol. 2014;32(13):1365-83. doi: 10.1200/JCO.2013.54.1177.

6. Rutgers EJ. Guidelines to assure quality in breast cancer surgery. Eur J Surg Oncol. 2005;31(6):568-76. PMID: 16023942.

7. Straver ME, Meijnen P, van Tienhoven G, van de Velde CJ, Mansel RE, Bogaerts J, Duez N, Cataliotti L, Klinkenbijl JH, Westenberg HA, van der Mijle H, Snoj M, Hurkmans C, Rutgers EJ. Sentinel node identification rate and nodal involvement in the EORTC 10981-22023 AMAROS trial. Ann Surg Oncol. 2010;17(7):1854 61. doi: 10.1245/s10434-010-0945-z.

8. Thill M, Kurylcio A, Welter R, van Haasteren V, Grosse B, Berclaz G, Polkowski W, Hauser N. The Central-European SentiMag study: sentinel lymph node biopsy with superparamagnetic iron oxide (SPIO) vs. radioisotope. Breast. 2014;23(2):175-9. doi: 10.1016/j. breast.2014.01.004.

9. Kalimo K, Jansen CT, Kormano M. Sensitivity to Patent Blue dye during skin-prick testing and lymphography. A retrospective and prospective study. Radiology. 1981;141(2):365-7. doi: 10.1148/ radiology.141.2.7291558.

10. Mertes PM, Malinovsky JM, Mouton-Faivre C, Bonnet-Boyer MC, Benhaijoub A, Lavaud F, Valfrey J, O'Brien J, Pirat P, Lalourcey L, Demoly P. Anaphylaxis to dyes during the perioperative period: reports of 14 clinical cases. J Allergy Clin Immunol. 2008;122(2):348-52. doi: 10.1016/j.jaci.2008.04.040.

11. Haque RA, Wagner A, Whisken JA, Nasser SM, Ewan PW. Anaphylaxis to patent blue V: a case series and proposed diagnostic protocol. Allergy. 2010;65(3):396-400. PMID: 19930233.

12. Wohrl S, Focke M, Hinterhuber G, Stingl G, Binder M. Near-fatal anaphylaxis to patent blue V. Br J Dermatol. 2004;150(5):1037-8. doi: 10.1111/j.1365-2133.2004.05931.x.

13. Salhab M, Al Sarakbi W, Mokbel K. Skin and fat necrosis of the breast following methylene blue dye injection for sentinel node biopsy in a patient with breast cancer. Int Semin Surg Oncol. 2005;2:26. doi: 10.1186/1477-7800-2-26.

14. Scherer K, Studer W, Figueiredo V, Bircher AJ. Anaphylaxis to isosulfan blue and cross-reactivity to patent blue $\mathrm{V}$ : case report and review of the nomenclature of vital blue dyes. Ann Allergy Asthma Immunol. 2006;96(3):497-500. doi: 10.1016/S10811206(10)60921-0.

15. Pinheiro LG, Oliveira Filho RS, Vasques PH, Filgueira PH, Aragao DH, Barbosa PM, Beserra HE, Cavalcante RV. Hemosiderin: a new marker for sentinel lymph node identification. Acta Cir Bras. 2009;24(6):432-6. PMID: 20011826.

16. Sleth JC. [Anaphylaxis due to patent blue. Time to change dye?]. Ann Fr Anesth Reanim. 2008;27(6):515. doi: 10.1016/j. annfar.2008.05.004

17. Barthelmes L, Goyal A, Newcombe RG, McNeill F, Mansel RE, New S, groups As. Adverse reactions to patent blue V dye - The NEW START and ALMANAC experience. Eur J Surg Oncol. 2010;36(4):399-403. doi: 10.1016/j.ejso.2009.10.007.

18. Manson AL, Juneja R, Self R, Farquhar-Smith P, Macneill F, Seneviratne SL. Anaphylaxis to Patent Blue V: a case series. Asia Pac Allergy. 2012;2(1):86-9. doi: 10.5415/apallergy.2012.2.1.86.

19. Lanitis S, Filippakis G, Sidhu V, Al Mufti R, Lee TH, Hadjiminas DJ. Atypical anaphylactic reaction to Patent Blue during sentinel lymph node biopsy for breast cancer. Ann R Coll Surg Engl. 2008;90(4):338-9. doi: 10.1308/003588408X285702.

20. Ramin S, Azar FP, Malihe H. Methylene blue as the safest blue dye for sentinel node mapping: emphasis on anaphylaxis reaction. Acta Oncol. 2011;50(5):729-31. doi: 10.3109/0284186X.2011.562918.

21. Govaert GA, Oostenbroek RJ, Plaisier PW. Prolonged skin staining after intradermal use of patent blue in sentinel lymph node biopsy for breast cancer. Eur J Surg Oncol. 2005;31(4):373-5. doi: 10.1016/j. ejso.2004.12.009.

22. Gumus M, Gumus H, Jones SE, Jones PA, Sever AR, Weeks J. How long will I be blue? Prolonged skin staining following sentinel lymph node biopsy using intradermal patent blue dye. Breast Care (Basel). 2013;8(3):199-202. doi: 10.1159/000352092.

23. Masannat YA, Hanby A, Horgan K, Hardie LJ. DNA damaging effects of the dyes used in sentinel node biopsy: possible implications for clinical practice. J Surg Res. 2009;154(2):234-8. doi: 10.1016/j. jss.2008.07.039.

\section{Correspondence:}

Luiz Gonzaga Porto Pinheiro

Departamento de Cirurgia - Universidade Federal do Ceará (UFC)

Rua Professor Costa Mendes, 1608/ $3^{\circ}$ andar

60430-140 Fortaleza-CE Brasil

Tel.: (55 85)3366-8063

Fax: (55 85)3283-7851

luizgporto@uol.com.br

Received: July 10, 2015

Review: Sep 11, 2015

Accepted: Oct 13, 2015

Conflict of interest: none

Financial source: Banco do Nordeste do Brasil S.A.

${ }^{1}$ Research performed at Grupo de Educação e Estudos Oncológicos (GEEON), Extension of the Surgery Department, Universidade Federal do Ceará (UFC), Brazil. 